\title{
Numerical calculations of diffraction losses in advanced interferometric gravitational wave detectors
}

\author{
Pablo Barriga \\ School of Physics, The University of Western Australia, Crawley, Western Australia 6009, Australia \\ Biplab Bhawal \\ Laser Interferometer Gravitational Wave Observatory Laboratory, California Institute of Technology, Pasadena, \\ California 91125, USA \\ Li Ju and David G. Blair \\ School of Physics, The University of Western Australia, Crawley, Western Australia 6009, Australia \\ Received October 3, 2006; accepted November 29, 2006; \\ posted December 18, 2006 (Doc. ID 75705); published May 9, 2007

\begin{abstract}
Knowledge of the diffraction losses in higher-order modes of large optical cavities is essential for predicting three-mode parametric photon-phonon scattering, which can lead to mechanical instabilities in long-baseline gravitational wave detectors. We explore different numerical methods in order to determine the diffraction but also influence the shape and frequency of the mode, which ultimately affect the parametric instability gain. Results depend on both the optical mode shape (order) and the mirror diameter. We also present a physical
\end{abstract} \\ losses of the higher-order optical modes. Diffraction losses not only affect the power buildup inside the cavity \\ interpretation of these results. (c) 2007 Optical Society of America \\ OCIS codes: $050.1940,120.2230,120.3180$.
}

\section{INTRODUCTION}

To detect gravitational waves, large laser interferometers have been built with arms formed by Fabry-Perot cavities stretching up to $4 \mathrm{~km}$. The actual interferometers are very close to their design sensitivity, but this may still not be enough to detect gravitational waves. To increase their sensitivity, advanced laser interferometer gravitational wave detectors will require much higher circulating optical power. The high power increases the high-frequency $(>100 \mathrm{~Hz})$ sensitivity but also enhances undesired effects including the possibility of parametric instability, which was first predicted by Braginsky et al. ${ }^{1}$ with further study by Kells and D'Ambrosio ${ }^{2}$ and Zhao et al. ${ }^{3}$

Parametric instabilities in advanced gravitational wave interferometers are predicted to arise because of a three-mode optoacoustic resonant scattering process in which the cavity fundamental mode $w_{0}$ scatters with test mass acoustic modes $w_{m}$ and optical cavity modes $w_{1}$, which satisfy the condition $w_{0} \sim w_{m}+w_{1}$. The parametric gain $R_{0}$ for this process determines whether the system is stable $\left(R_{0}<1\right)$ or unstable $\left(R_{0} \geq 1\right)$. Three-mode optoacoustic parametric processes have not yet been observed. However, two-mode processes that also couple optical and mechanical degrees of freedom via radiation pressure have been observed in resonant bar gravitational wave detectors with microwave resonator readouts. ${ }^{4}$ More recently they have been observed in optical microcavities with very high $Q^{5}$ and low frequencies in a short $(\sim 1 \mathrm{~m})$ suspended optical cavity. ${ }^{6}$ In all these cases the mechanical mode frequency is within the electromagnetic mode linewidth.

It has been shown that for an Advanced Laser Interferometer Gravitational Wave Observatory (LIGO) type of interferometer with fused-silica test masses the parametric gain $R_{0}$ will typically have a value of $\sim 10$ (see Ref. 7 ). The parametric gain scales directly as the mechanical $Q$ factor of the test masses and the optical $Q$ factor of higher-order modes. Hence errors in the $Q$ factor of higher-order modes directly affect the estimation of $R_{0}$. Thus it is very important to have an accurate estimation of the diffraction losses of the modes. Equation (1) shows the parametric gain in a power-recycled interferometer:

$$
R_{0} \approx \frac{2 P Q_{m}}{M c L w_{m}^{2}}\left(\frac{Q_{1} \Lambda_{1}}{1+\Delta w_{1}^{2} / \delta_{1}^{2}}-\frac{Q_{1 a} \Lambda_{1 a}}{1+\Delta w_{1 a}^{2} / \delta_{1 a}^{2}}\right) .
$$

Here $P$ is the total power inside the cavity, $M$ is the mass of the test mass, $Q_{1(a)}$ are the quality factors of the Stokes (anti-Stokes) modes, $Q_{m}$ is the quality factor of the acoustic mode, $\delta_{1(a)}=w_{1(a)} / 2 Q_{1(a)}$ corresponds to the relaxation rate, $L$ is the cavity length, $\Delta w_{1(a)}=w_{0}-w_{1(a)}-w_{m}$ is the possible detuning from the ideal resonance case, and $\Lambda_{1}$ 
and $\Lambda_{1 a}$ are the overlap factors between optical and acoustic modes. The overlap factor is defined as ${ }^{1}$

$$
\Lambda_{1(a)}=\frac{V\left(\int f_{0}\left(\vec{r}_{\perp}\right) f_{1(a)}\left(\vec{r}_{\perp}\right) u_{z} \mathrm{~d} \vec{r}_{\perp}\right)^{2}}{\int\left|f_{0}\right|^{2} \mathrm{~d} \vec{r}_{\perp} \int\left|f_{1(a)}\right|^{2} \mathrm{~d} \vec{r}_{\perp} \int|\vec{u}|^{2} \mathrm{~d} V} .
$$

Here $f_{0}$ and $f_{1(a)}$ describe the optical field distribution over the mirror surface for the fundamental and Stokes (antiStokes) modes, respectively, $\vec{u}$ is the spatial displacement vector for the mechanical mode, and $u_{z}$ is the component normal to the mirror surface. The integrals $\int \mathrm{d} \vec{r}_{\perp}$ and $\int \mathrm{d} V$ correspond to integration over the mirror surface and mirror volume, respectively.

Traditionally, it has been assumed that diffraction losses can be estimated by the clipping approximation. In this approximation it is assumed that the mode shape is not altered by the finite-mirror geometry and that the diffraction loss is simply determined by the fraction of the mode that overlaps the mirror.

It has been demonstrated, for the fundamental mode of a two-mirror symmetric cavity, that the geometry of the system determines whether the clipping approximation overestimates or underestimates the diffraction losses. ${ }^{8,9}$ Preliminary work by D'Ambrosio ${ }^{10}$ indicates that the clipping approximation is not valid for this particular case. For this reason we have undertaken careful numerical modeling of the mode losses based on the free propagation of the beams inside a long-baseline interferometer. Using fast Fourier transform (FFT) simulations, we determine the diffraction losses inside the main arms of a proposed advanced interferometer configuration. We present results for typical arm cavity design for advanced interferometers, based on the proposed design of Advanced LIGO.

We show that for these very large cavities the mode frequencies are shifted by a significant amount and that the size of the mirror not only affects the diffraction losses but also the cavity gain, mode frequency, $Q$ factor, and the mode shape. All these parameters are necessary to determine the overlap factor $\Lambda$ and greatly affect the predicted parametric gain $R_{0}$.

First, we introduce the FFT simulation method to simulate the behavior of a Gaussian beam inside a cavity. In the next section this is applied to calculate the diffraction losses from Hermite-Gaussian (HG) and LaguerreGaussian (LG) modes in an advanced gravitational wave detector arm cavity, analyzing also the limits of the method. In Section 4 these results are compared with results from eigenvalue simulations. We also analyze the change in diffraction losses when using finite mirrors of different sizes, including their impact on the optical gain and mode frequency, the optical $Q$ factor, and mode shape.

\section{DIFFRACTION LOSSES}

Diffraction losses occur in any optical cavity with finitesize optics, even if the mirrors are large compared with the Gaussian spot size. The larger the Fresnel number, the weaker is the field intensity at the edge of the mirror and the smaller the power loss due to diffraction loss. ${ }^{11,12}$
If we consider a Fabry-Perot cavity (such as the proposed Advanced LIGO arm cavities) with mirrors of diameter $2 a$ and spot size radius $\omega$, we can say that only those modes of order less than the ratio of the two areas will oscillate inside the cavity with relatively low losses ${ }^{13}$ :

$$
N_{\text {max }} \approx\left(\frac{\pi a^{2}}{\pi w^{2}}\right) \text {. }
$$

We use the proposed design of Advanced LIGO as a case in point. It has mirrors of $34 \mathrm{~cm}$ diameter with a spot size of approximately $6 \mathrm{~cm}$, which gives us $N_{\max }=8.03$. Therefore we will analyze modes up to the eighth order. We can expect that the cavity losses for higher-order modes will rapidly increase with mode number.

The clipping approximation determines the part of the higher-order mode spot size that will fall outside the mirror's surface when the mode shape itself is due to that of an infinite-diameter mirror. The diffraction loss in each reflection of a cavity mode off a mirror is given by

$$
\mathcal{D}_{\text {clip }}=\int_{a}^{\infty}|U(r)|^{2} 2 \pi \mathrm{d} r .
$$

Here $U(r)$ is the normalized field of a HG or LG mode with infinite-size mirrors integrated outside a mirror of diameter $2 a$.

It is already known that the clipping approximation yields a smaller loss than the calculations based on FFT methods for the $\mathrm{TEM}_{00}$ mode in a long optical cavity. The FFT method enables the mode shape changes due to the finite mirror sizes to be estimated and hence enables a much better approximation of the diffraction losses. A good explanation of this method can be found in Ref. 14 and a more general explanation in Ref. 15.

The following calculation relates the internal power to the diffraction losses due to finite-size mirrors. Let $T_{i}, D_{i}$, and $L_{i}$ be the transmission, diffraction, and dielectric losses, respectively, for the input test mass (ITM) and $T_{e}$, $D_{e}$, and $L_{e}$ be the corresponding values for the end test mass (ETM). The finesse of such cavity can then be calculated as $^{16}$

$$
\mathcal{F} \cong \frac{2 \pi}{T_{i}+D_{i}+L_{i}+T_{e}+D_{e}+L_{e}} .
$$

Since we are interested in the effects of the diffraction losses over the circulating power inside the cavity in steady state, we assume perfect mode matching for the input beam. Therefore the peak value for the circulating intensity in a purely passive cavity at resonance can be written as $^{17}$

$$
I_{c i r c} \approx \frac{4\left(T_{i}+L_{i}\right)}{\left(T_{i}+D_{i}+L_{i}+T_{e}+D_{e}+L_{e}\right)^{2}} I_{i n c}
$$

In the case of infinite-size mirrors there are no diffraction losses; then $D_{i}=D_{e}=0$.

For our simulations we use the following values, which coincide with proposed parameters for Advanced LIGO interferometers, where $T_{i}=5000 \mathrm{ppm}$ (parts per million), 
$T_{e}=1 \mathrm{ppm}$, and $L_{i}=L_{e}=15 \mathrm{ppm} .{ }^{18}$ In this case it is clear that the major loss contribution comes from the transmission losses of the ITM.

Note that Eq. (6) does not contain a mode-matching parameter because of the assumption of perfect mode matching. This assumption allows us to refer the incident beam to the cavity waist; this simplifies the analysis.

To calculate the diffraction losses, we use a lossless cavity in parallel with the cavity under study. After each round trip the resulting beam is normalized and propagated into a lossless cavity; this cavity has the same characteristics of the cavity under study but with lossless mirrors. In such cavity, diffraction losses are the only cause of power loss. Therefore we can calculate the diffraction losses per round trip using Eq. (7), where $P_{j}^{\text {Norm }}$ corresponds to the normalized total circulating power per round trip:

$$
\mathcal{D}_{\text {clip }}=1-P_{j}^{\text {Norm }} \text {. }
$$

In the case of an infinite-sized mirror, $P_{j}^{\text {Norm }}$ is always 1 , hence no diffraction losses. This method proved to be more accurate than measuring the diffraction losses on every round trip using the incremental power of every round trip as shown in Eq. (8):

$$
\mathcal{D}=\left(1-\frac{p_{j}}{p_{j-1}}\right)-\left(T_{i}+L_{i}+T_{e}+L_{e}\right) .
$$

Here $p_{j}$ corresponds to the power contribution of round trip $j$. Therefore $p_{j-1}$ corresponds to the power contribution of the previous round trip. This method is more susceptible to numerical errors due to the digitization of the beam.

A third way to estimate the diffraction losses is to calculate the eigenvalues for the cavity. At the end of Subsection 4.A we comment on the verification of our results based on comparison with eigenvalue calculations. Eigenvalues $\gamma_{m n}$ are such that after one round trip the eigenmodes will satisfy the simplified round-trip propagation expression $^{19}$

$$
\gamma_{m n} U_{m n}(x, y)=\iint K\left(x, y, x_{0}, y_{0}\right) U_{m n}\left(x_{0}, y_{0}\right) \mathrm{d} x_{0} \mathrm{~d} y_{0} .
$$

The magnitude of the eigenvalues due to the round-trip losses will be less than unity. Therefore we can calculate the power loss per round trip as

$$
\text { Power loss per round trip }=1-\left|\gamma_{m n}\right|^{2} \text {. }
$$

We now go on to present the details of our FFT simulation methods.

\section{FFT SIMULATION}

Since each mode has a different resonant frequency depending on the order of the mode, we must analyze each mode separately. The first step is to generate the mode we are interested in. Part of the study also includes the comparison between HG and LG modes. For simplicity the mode is generated at the waist of the cavity. HG modes are given by

$$
\begin{aligned}
U_{m, n}(x, y, z)= & \left(\frac{1}{2 \pi}\right)^{1 / 2} \sqrt{\frac{\exp [j(2 m+2 n+1) \psi(z)]}{2^{m} 2^{n} m ! n ! w(z)^{2}}} \\
& \times H_{m}\left[\frac{\sqrt{2 x}}{w(z)}\right] H_{n}\left[\frac{\sqrt{2} y}{w(z)}\right] \\
& \times \exp \left\{-j 2 k z-j k\left[\frac{x^{2}+y^{2}}{2 R(z)}\right]-\frac{x^{2}+y^{2}}{w(z)^{2}}\right\} .
\end{aligned}
$$

Here $m$ and $n$ correspond to the order of the transverse modes, $w(z)$ is the spot size radius, $R(z)$ is the beam radius of curvature, $\psi(z)$ is the Gouy phase shift, $k$ is the wavenumber, and $H_{m}()$ is the $m$ th-order Hermite polynomial.

LG modes are also a valid representation of the higherorder modes, this time in cylindrical coordinates rather than rectangular, and are given by

$$
\begin{aligned}
U_{l, m}(r, \phi, z)= & \sqrt{\frac{4 l !}{\left(1+\delta_{0, m}\right) \pi(l+m) !}} \\
& \times\left\{\frac{\exp [j(2 l+m+1) \psi(z)]}{w(z)}\right\} \\
& \times \cos (m \phi)\left[\frac{\sqrt{2} r}{w(z)}\right]^{m} L_{l, m}\left[\frac{2 r^{2}}{w(z)^{2}}\right] \\
& \times \exp \left[-j k z-j z \frac{r^{2}}{2 R(z)}-\frac{r^{2}}{w(z)^{2}}\right] .
\end{aligned}
$$

Here $l$ corresponds to the radial index and $m$ to the azimuthal mode index, $L_{l, m}()$ are the generalized Laguerre polynomials, $\delta_{0, m}=1$ if $m=0$, and $\delta_{0, m}=0$ if $m>0$. The rest of the variables are the same as in the HG modes.

The Fourier transform corresponds to the transformation of the beam profile into a spatial-frequency domain. We can create a propagation matrix based on an expansion of the optical beam in a set of infinite plane waves traveling in slightly different directions, ${ }^{20}$ given by

$$
A\left(p, q, z_{L}\right)=\exp \left[-j k z_{L}+j \pi \lambda\left(p^{2}+q^{2}\right) z_{L}\right],
$$

where $z_{L}$ corresponds to the distance that we will propagate the beam and $p$ and $q$ are the coordinates in the Fourier space or the spatial frequencies. To apply this propagation matrix, it is also necessary to transform the field of the input beam by using a two-dimensional FFT. The Fourier transform of a Gaussian function is always another Gaussian transform of the same order, i.e.,

$$
\mathbb{F}\{U(x, y, z)\}=\iint U(x, y, z) e^{-j p x} e^{-j q y} \mathrm{~d} x \mathrm{~d} y,
$$

and the inverse Fourier transform is then written as

$$
\mathbb{F}^{-1}\{U(p, q, z)\}=\left(\frac{1}{2 \pi}\right)^{2} \iint U(p, q, z) e^{j p x} e^{j q y} \mathrm{~d} p \mathrm{~d} q .
$$

Once the beam has been propagated by multiplying point to point both the field matrix and the propagation 
matrix, we can transform the field back to the time domain using the two-dimensional inverse FFT. If $z_{0}$ is the propagation starting point, then the final field corresponds to

$$
U\left(x, y, z_{L}\right)=\mathbb{F}^{-1}\left\{\mathrm{~F}\left[U\left(x, y, z_{0}\right)\right] \times A\left(p, q, z_{L}\right)\right\} .
$$

Thus, this method basically consists of transforming the input field into the Fourier domain in two dimensions and propagation of this field along the $z$ axis. Then the resulting field is transformed back to the time domain using the inverse FFT. Based on this principle, we developed our own code in MATLAB, which allowed us to propagate the field and to reflect it off the mirror surface. Two independent codes were developed, one at the University of Western Australia (UWA) and the other at the California Institute of Technology (Caltech), for the purpose of verification of results. For the simulation we assume a perfect surface for the test masses, but any imperfection can easily be added. Starting from the waist of the cavity, we propagate the beam down to the ETM where it is reflected from the mirror surface and propagate it back through the waist to the ITM. Here part of the beam is transmitted out of the cavity through the substrate, and the rest is reflected back to the waist of the cavity, completing the round trip. This is then iterated until the power inside the cavity has reached the steady state.

To calculate the diffraction losses for different higherorder modes, it was necessary first to make the modes resonant inside the cavity. Starting from a nominal value of $4 \mathrm{~km}$ for the cavity length, we move the ETM away from the ITM up to a maximum of half the laser wavelength $\left(\lambda=1.064 \times 10^{-6} \mathrm{~m}\right)$ until we find the cavity length that maximizes the circulating power for a particular mode. For each small step that the ETM is moved, several round trips are done to calculate the power built up inside the cavity. Once the cavity is set at the resonance length, we propagate the mode inside the cavity for several round trips until the circulating power reaches the steady state, which corresponds to the maximum circulating power.

Most of the calculations presented here were done using a $128 \times 128$ element grid. This proved to be good for the calculations of the parameters we are interested in, for the different modes. However, it was observed that for higher-order modes (order higher than 6) a grid of 128 $\times 128$ is not enough when mirrors of infinite size are used, due to aliasing. In those cases we increased the size of the grid to $256 \times 256$, using also finer elements. Another option could have been the use of antialiasing filters or an adaptive grid.

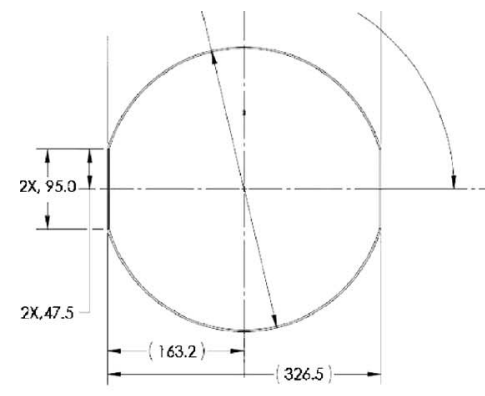

\section{RESULTS}

To determine the parametric instability gain, we need to know more than just the diffraction losses of the higherorder modes. Therefore we also calculated the optical gain, the frequency, and the optical $Q$ factor of each mode. We also examined the mode shapes and how they change with the size of the mirrors. In each case the calculations were done separately for each mode. We explored the variation of the size of the mirrors that form the cavity, while keeping the mirror radius of curvature and losses constant. The results were crossed checked between UWA and Caltech, with very good agreement between both simulations. A cavity formed by two mirrors with the losses previously mentioned and a constant radius of curvature of $2076 \mathrm{~m}$ at a nominal length of $4000 \mathrm{~m}$ was assumed for these simulations. On each simulation a pair of mirrors of the same diameter was used. For all our simulations it was assumed that the substrate of the test masses will have two flat sides proportional to the substrate diameter for suspension attachment as shown in Fig. 1. We also assumed that the mirror coating covers the whole front surface of the test mass (surface 1 in Fig. 1).

\section{A. Diffraction Losses}

The diffraction losses for different modes for varying mirror size are presented in Fig. 2. As expected, the diffraction losses are the same for modes of order 1 , such as HG01, HG10, and LG01. For HG modes the order number is given by $(m+n)$ while for LG modes the order number is given by $(2 l+m)$.

It is clear (as previously shown by Fox and Li in Ref. 11) that the higher the order of the mode, the higher is the loss, especially for smaller mirrors (smaller Fresnel number). Diffraction losses can be separately analyzed by the order of the mode, but when the order of the mode increases we need to take into account the symmetry of the modes. For example, mode HG60 is mainly distributed along one axis compared with HG33, which is of the same order but is evenly distributed on the mirror's surface. In such a case and due to the energy distribution of each mode the losses for mode HG33 are much smaller than those for mode HG60.

From Fig. 2 we can deduce that when the order of the mode is increased the grouping of the diffraction losses spreads out due to the different symmetries of the modes. If we compare the diffraction losses of modes of orders 6 and 7 [Figs. 2(c) and 2(d)], we notice that there is an overlap of some modes. Therefore the low-loss modes of order

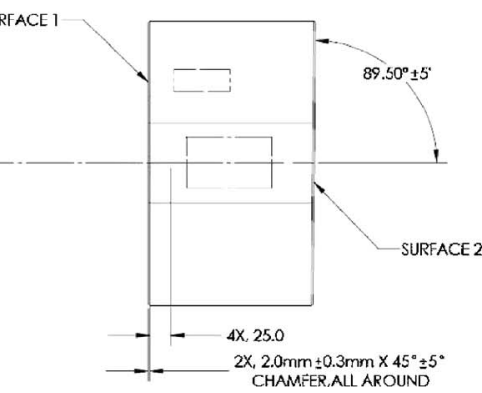

Fig. 1. Advanced LIGO substrate dimensions for ITM and ETM test masses showing the flat sides of the test mass for suspension attachment. From LIGO drawing D040431-B. 


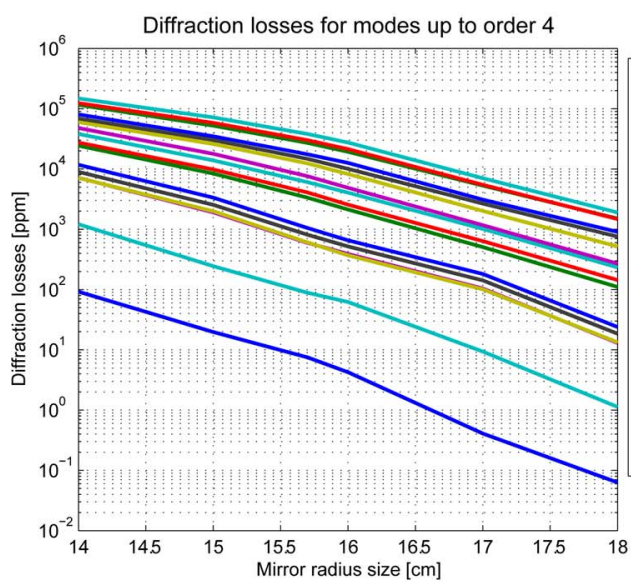

(a)

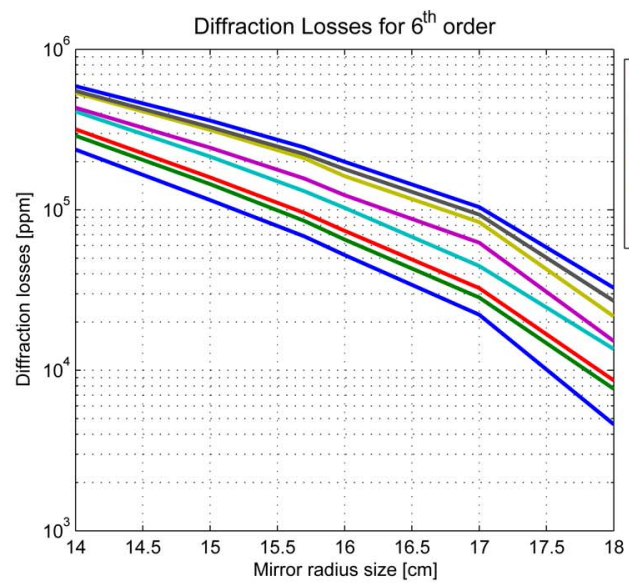

(c)

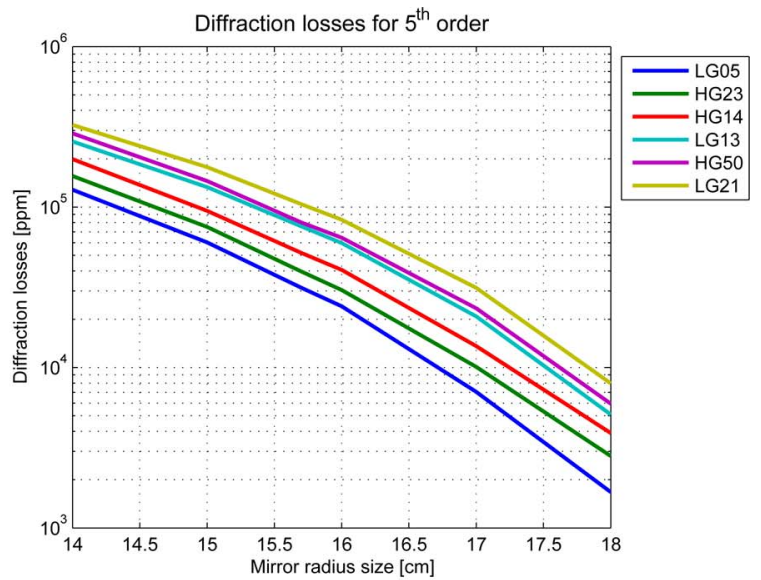

(b)

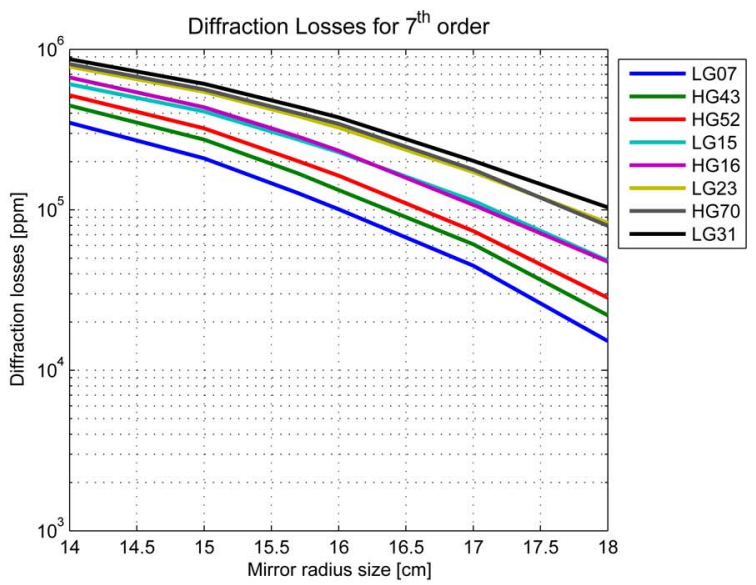

(d)

Fig. 2. (Color online) Diffraction losses for different higher-order modes. Starting from the top left corner, we present modes up to order 4 in ascendant orders from HG00 to LG20. For clarity we separately present modes of orders 5, 6, and 7. HG and LG modes are plotted together for comparison.
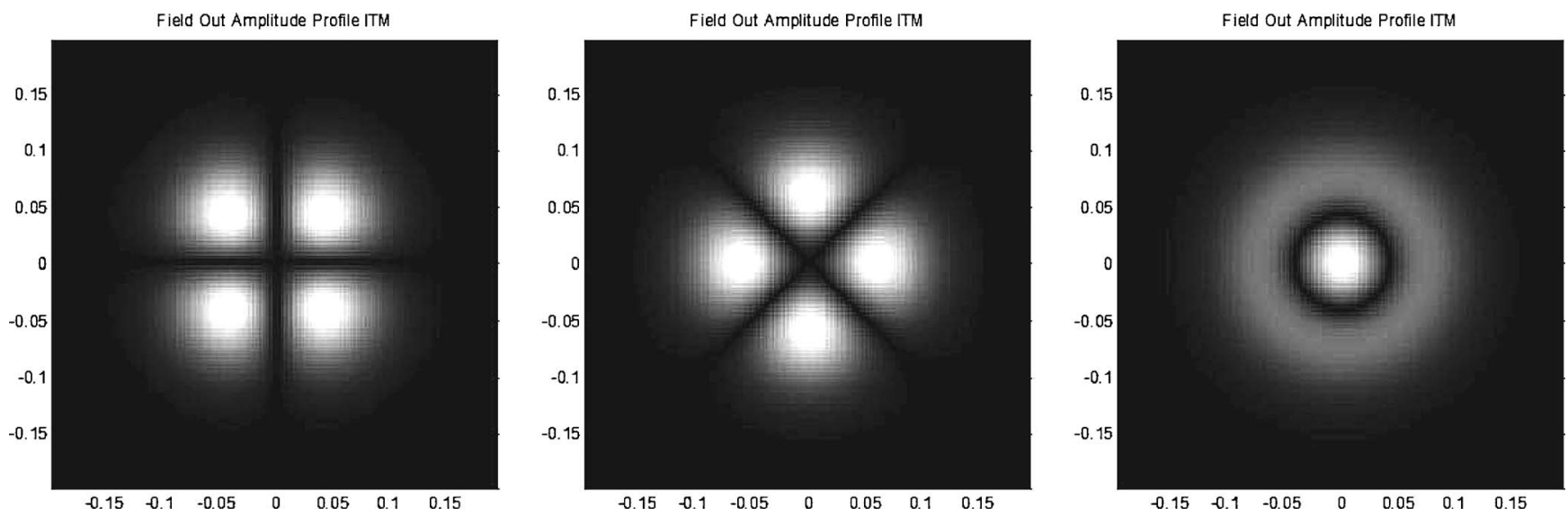

Fig. 3. Intensity profile at the ITM in a mirror of diameter $34 \mathrm{~cm}$. On the left-hand side we can see mode HG11, compared with mode LG02 in the middle. Also for comparison the energy distribution of mode LG10 is presented on the right-hand side.

7 such as LG07 have lower diffraction losses than the high-loss modes of order 6, namely, LG22 and LG30.

Even though it is difficult to distinguish in Fig. 2, modes HG11 and LG02 have almost the same losses. This is easy to explain, since we can see in Fig. 3 that LG02 corresponds to HG11 twisted by $45 \mathrm{deg}$ (or vice versa), and they are therefore orthogonal to each other. Therefore the difference in diffraction losses comes from the fiat sides of the test mass affecting differently each mode. It is also interesting to notice that the highest loss is in mode 
LG10, which is a more symmetric one but has more energy at the edge of the mirror, compared with other second-order modes.

We have compared our results with calculations of the diffraction losses using the cavity eigenvalues by Agresti from Caltech. ${ }^{21} \mathrm{He}$ calculated the diffraction losses for several LG modes. His results are in very close agreement with the results here presented. The average difference between the FFT simulation and the eigenvalue approximation is of $1 \%$, while the biggest difference is less than $5 \%$ for lower-order modes. However, his calculations were done for a previous design of Advanced LIGO test masses with smaller mirrors of $31.4 \mathrm{~cm}$ in diameter, which we also use for comparison.

In parallel with the FFT simulations and the eigenvalue calculations done at Caltech, we did our own eigenvalue calculations based on an eigenvector method proposed by Yuanying et al. ${ }^{22}$ The results obtained through this method showed that in a perfectly aligned cavity with cylindrical test masses (circular mirrors) only LG modes and their rotated orthogonal modes will resonate. However, the need to suspend the mirrors requires the test masses to have two flat sides, as can be seen in Fig. 1 (LIGO Technical Document D040431-B). This breaks the symmetry.

As the circular symmetry is broken when solving with the eigenvector method for this cavity, it shows that HG modes are now part of the eigenvector solution of this cavity. Therefore HG modes are partially supported by the cavity even if it is perfectly aligned. However, these modes are mainly distributed along the horizontal axis aligned with the flat sides of the test mass. The symmetry break not only changes the eigenvector and eigenvalue solution for this system but also induces a particular orientation of the higher-order mode that minimizes the diffraction losses.

The main difference with the eigenvector method is that in the FFT simulation we can choose which mode we are going to propagate inside the cavity. The FFT simulation allows the mode to change its shape while it propagates inside the cavity. To determine when the mode shape is stable, we calculate the nonorthogonality between the input and the circulating beam, this subject to the finesse of the mode and the power to build up in the cavity. Therefore the mode labels in the graphs correspond to the input mode used in the FFT simulation and do not necessarily correspond to that of the final mode shape.

Figure 4 shows the diffraction loss results obtained with both methods. The results are in close agreement, but we noticed that some of the modes that we injected are not supported by the cavity. These modes, in fact, do not appear in the eigenvector method; moreover, we can see that those modes are the ones in which their mode shape changes into a lower-loss mode of the same order. This explains why modes HG15, HG24, and HG33 all have losses similar to those of mode LG06. We further analyze the mode shape changes in Subsection 4.D.

\section{B. Optical Gain}

Considering the parameters here presented for Advanced LIGO-type optical cavities, the optical gain of the main arms is about 800. Assuming infinite-size mirrors and the losses already presented for this cavity, the gain that we obtain from the simulations is close to 793. Using mirrors of finite size increases the diffraction losses, thus reducing the gain. This can also be deduced from Eq. (6).

For a given finite mirror size the higher the order of the mode, the higher the diffraction losses. This in turn means lower optical gain and lower finesse for the higherorder mode, which as a consequence implies a reduction of the circulating power. Figure 5 shows the optical gain for some of the HG and LG modes. The figure shows how the gain of each order changes with the mirror size (or Fresnel number). The fundamental mode does not change much, but as the order of the mode increases the diffrac-

Diffraction losses comparison between FFT and Eigenvalues

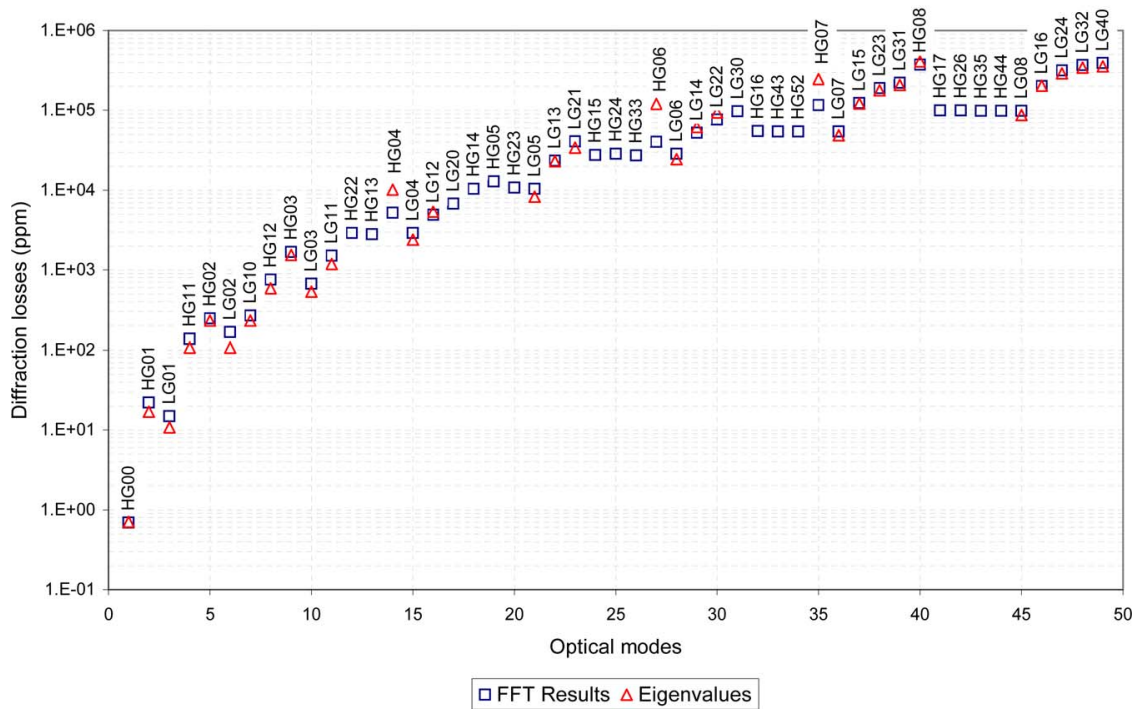

Fig. 4. (Color online) Comparison of diffraction losses obtained with FFT simulations and the eigenvector method for the proposed Advanced LIGO-type cavity. 
tion losses increase, reducing the gain. With infinite-size mirrors, all the modes have the same gain, since there are no diffraction losses.

Using the nominal Advanced LIGO mirror size $(34 \mathrm{~cm}$ diameter), we can plot the optical gain versus mode number as shown in Fig. 6. Here we notice how the gain is reduced for a particular mirror size when the order of the mode increases. Again, we can see the dependency on the energy distribution and symmetry of the mode.

The gain reduction due to the finite size of the mirrors can also be appreciated in Fig. 7, which shows the effect in the intensity profile of mode HG40. We can see how the optical gain is reduced with the size of the mirrors. The use of smaller mirrors increases the diffraction losses, leading to smaller gain as shown in Table 1 . This effect is even stronger in higher-order modes.

\section{Mode Frequency}

As previously mentioned, the calculation of the resonance length was done by moving the ETM away from the ITM

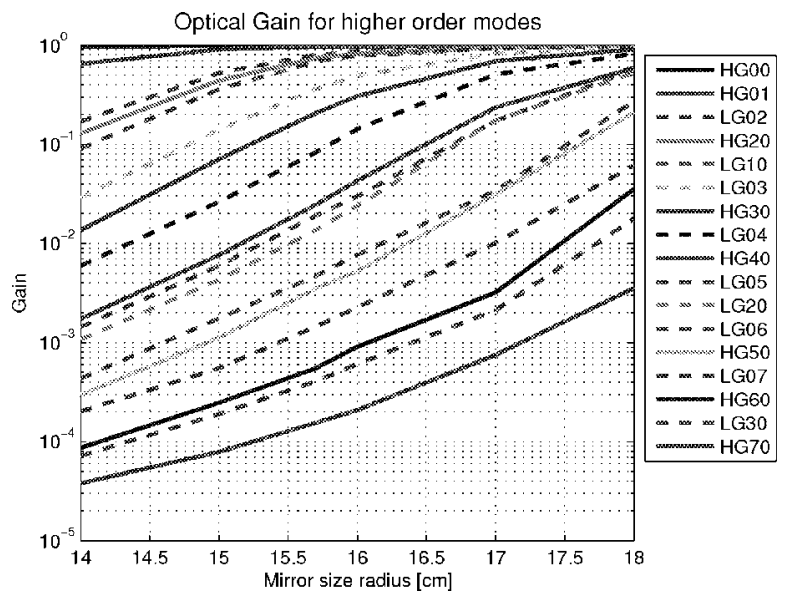

Fig. 5. (Color online) Cavity optical gain for some HG modes of different orders. The modes have been plotted in descendant order, starting from the top with HG00 to finish with HG70 at the bottom. HG and LG modes are plotted together for comparison. until the circulating power is maximized. The resonance length is different for each mode, but when the mirror size was changed a minor variation in resonance length was noticed. This suggests that mirrors of different sizes will also alter the resonance conditions of the cavity, thus changing the mode frequency. It is well known that the frequency shift for higher-order modes is given by ${ }^{23}$

$$
\begin{aligned}
& \frac{v_{0}}{\pi}(m+n) \arccos \left(\sqrt{g_{1} g_{2}}\right) \text { for HG modes, } \\
& \frac{v_{0}}{\pi}(2 l+m) \arccos \left(\sqrt{g_{1} g_{2}}\right) \text { for LG modes. }
\end{aligned}
$$

Here $v_{0}$ corresponds to the free spectral range in hertz and $g_{1}$ and $g_{2}$ correspond to the stability factor of each mirror, defined as $g=(1-L / R), L$ being the cavity length and $R$ being the radius of curvature of the mirror. Thus to calculate the frequency separation of a higher-order mode

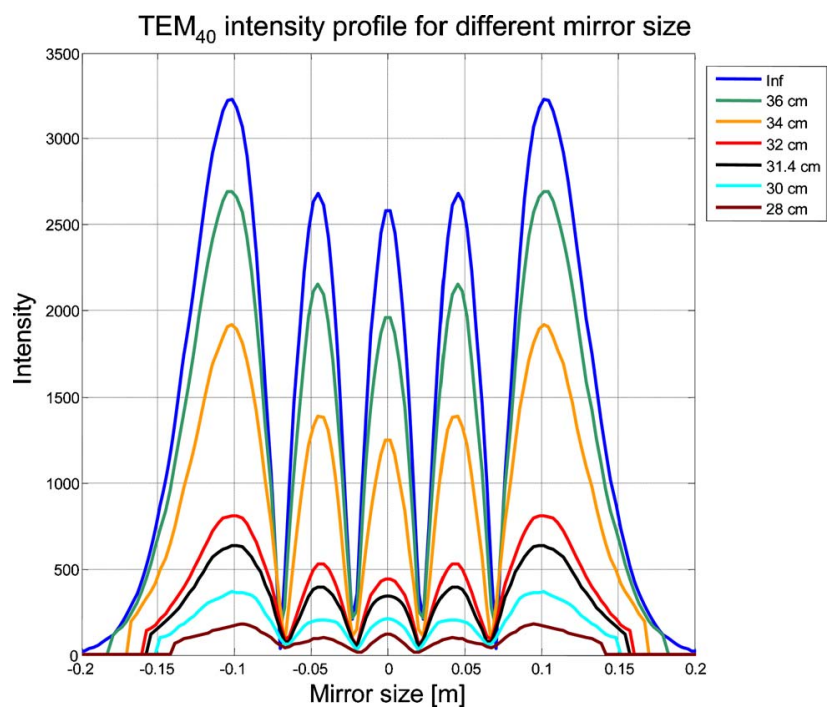

Fig. 7. (Color online) Intensity profile variation of mode HG40 due to different mirror sizes.

Higher-Order Modes Optical Gain

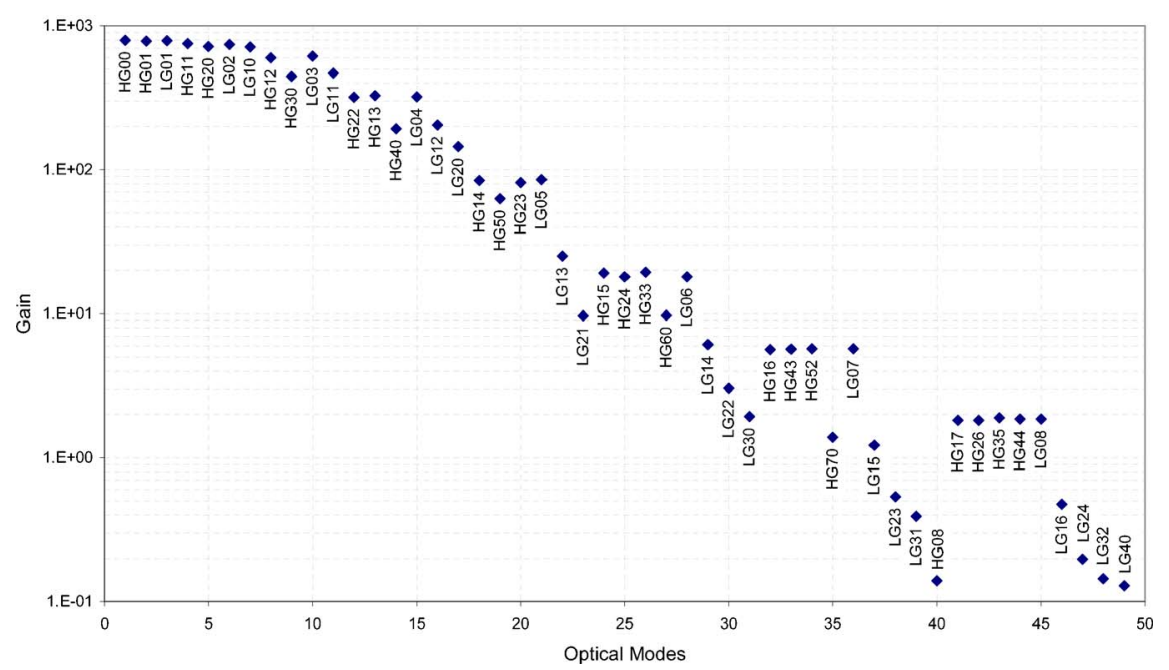

Fig. 6. (Color online) Optical gain variation for higher-order modes for a mirror diameter of $34 \mathrm{~cm}$. 
with respect to the fundamental mode, we use the following relation:

$$
\left|\frac{\Delta f}{\Delta l}\right|=\frac{1}{L} f_{Y A G}+\frac{N}{\pi} \frac{c}{2 L^{2}} \frac{1}{\left(2 L R-L^{2}\right)^{1 / 2}},
$$

where $\Delta f$ corresponds to the frequency variation of the mode, $\Delta l$ is the cavity length variation, and $f_{Y A G}$ is the $\mathrm{Nd}$ :YAG laser fundamental mode frequency. Here $N$ $=(m+n+1)$ for HG modes, $N=(2 l+m+1)$ for LG modes, $c$ corresponds to the speed of light in vacuum, and $R$ is the radius of curvature of the mirrors. We note that in reality the laser is locked to the $\mathrm{TEM}_{00}$ cavity mode. Equation (19) is a special case for cavities with two mirrors of the same radius of curvature and as a consequence with $g_{1}$ $=g_{2}$.

Using expressions (17) and (18), the mode separation per mode order using the proposed Advanced LIGO parameters is $4.593 \mathrm{kHz}$. According to our simulations, this is true only when mirrors of infinite size are used. The use of mirrors of finite size introduces diffraction losses that not only reduce the circulating power but also shift the frequency of the higher-order modes.

Table 1. Diffraction Losses and Cavity Optical Gain of Mode HG40 for Different Mirror Sizes

\begin{tabular}{ccc}
\hline $\begin{array}{c}\text { Mirror Diameter } \\
(\mathrm{cm})\end{array}$ & $\begin{array}{c}\text { Diffraction Losses } \\
(\mathrm{ppm})\end{array}$ & Optical Gain \\
\hline 28 & 116022 & 1.37 \\
30 & 52437 & 6.07 \\
32 & 19256 & 34.01 \\
34 & 5315 & 187.40 \\
36 & 1506 & 469.50 \\
\hline
\end{tabular}

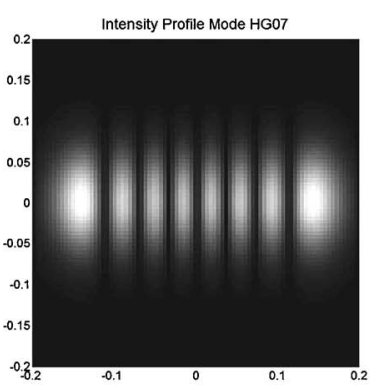

(a)

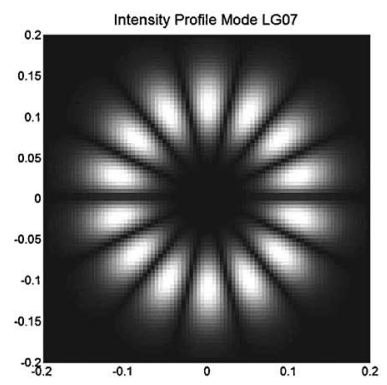

(e)

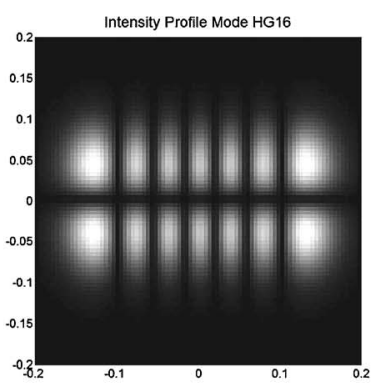

(b)

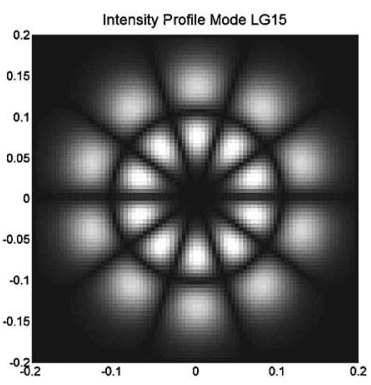

(f)

First we consider the fundamental mode where the frequency deviations are negligible, of the order of millihertz. The smallest mirror in our simulations is $28 \mathrm{~cm}$ in diameter. In this case the frequency variation from the infinite-mirror case is about $24 \times 10^{-3} \mathrm{~Hz}$, which, compared with the laser fundamental mode frequency is a variation of $10^{-17}$. For a mirror of $34 \mathrm{~cm}$ in diameter the frequency variation is reduced to $1.9 \times 10^{-3} \mathrm{~Hz}$.

These results show clear agreement with the diffraction losses for modes of order 7 shown in Fig. 2(d). The optional modes are shown in Fig. 8, and their frequency shift is shown in Table 2. The highest diffraction losses are from mode LG31, while the lowest losses are from mode LG07, which also has the smallest frequency variations from the infinite-mirror case. These results show that the frequency depends not only on the mode order but also on the symmetry and energy distribution of the mode subject to the size of the mirror. The frequency variation will also have an impact on the possible parametric instability calculations. Figure 9 shows the frequency variations for the higher-order modes in the proposed cavity with mirrors of $34 \mathrm{~cm}$ in diameter.

Cavity losses define the optical $Q$ factor, which for any given mode is given by

$$
Q=\frac{w}{2 \delta_{T}}
$$

Here $w$ corresponds to the frequency of the mode and $\delta_{T}$ corresponds to the relaxation rate of that particular mode. ${ }^{1}$ Therefore it is expected that the higher the diffraction losses, the lower the optical $Q$ of that mode. The $Q$ factor of the optical modes has a direct effect on the parametric gain $R_{0}$; therefore a reduction of this factor will also reduce the parametric gain. The $Q$ factor of the

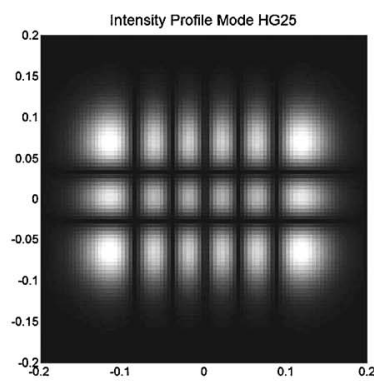

(c)

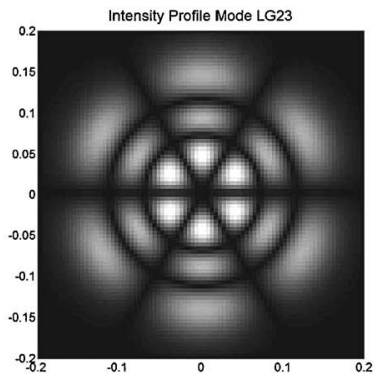

(g)

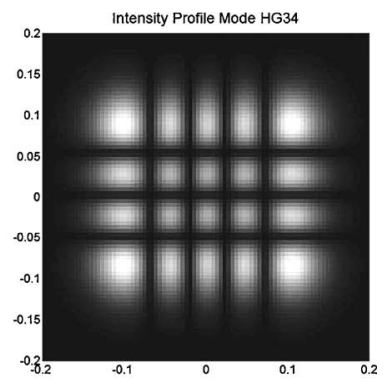

(d)

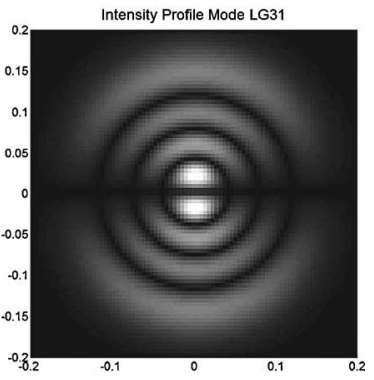

(h)

Fig. 8. Modes of order 7 as they will look in an infinite-size mirror. Therefore no diffraction losses or mode shape changes affect these modes. (a)-(d) Modes HG07, HG16, HG25, and HG34; (e)-(h) modes LG07, LG15, LG23, and LG31. 
Table 2. Frequency Shift of Modes of Order 7 for Different-Size Mirrors Compared with the Frequency of the Same Mode with Use of Infinite-Size Mirrors

\begin{tabular}{ccrrrrrrr}
\hline & \multicolumn{1}{c}{$\Delta$ freq } \\
\cline { 2 - 8 } $\begin{array}{c}\text { Mirror } \\
\text { Diameter }(\mathrm{cm})\end{array}$ & $\begin{array}{c}\text { HG70 } \\
(\mathrm{Hz})\end{array}$ & $\begin{array}{c}\text { HG61 } \\
(\mathrm{Hz})\end{array}$ & $\begin{array}{c}\text { HG52 } \\
(\mathrm{Hz})\end{array}$ & $\begin{array}{c}\text { HG43 } \\
(\mathrm{Hz})\end{array}$ & $\begin{array}{c}\text { LG07 } \\
(\mathrm{Hz})\end{array}$ & $\begin{array}{c}\text { LG15 } \\
(\mathrm{Hz})\end{array}$ & $\begin{array}{c}\text { LG23 } \\
(\mathrm{Hz})\end{array}$ & $\begin{array}{c}\text { LG31 } \\
(\mathrm{Hz})\end{array}$ \\
\hline 28 & 3466 & 1658 & 1345 & 1277 & 1251 & 2715 & 3735 & 4213 \\
30 & 2144 & 799 & 686 & 659 & 649 & 1584 & 2324 & 2698 \\
32 & 897 & 282 & 248 & 237 & 233 & 688 & 1127 & 1371 \\
34 & 317 & 103 & 70 & 64 & 61 & 215 & 400 & 515 \\
36 & 73 & 59 & 34 & 25 & 21 & 76 & 145 & 189 \\
\hline
\end{tabular}

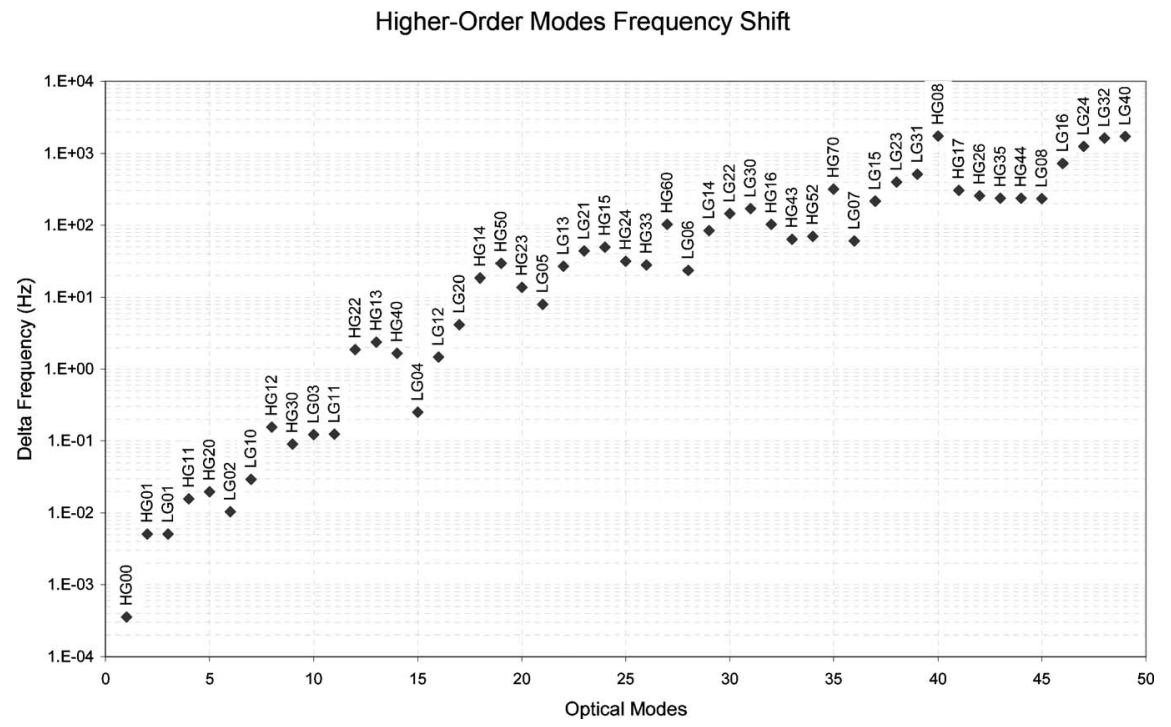

Fig. 9. Frequency variations from the theoretical value (infinite-size mirror) for each higher-order mode when finite-size mirrors of diameter $34 \mathrm{~cm}$ are used.

Optical Q factor for higher-order modes

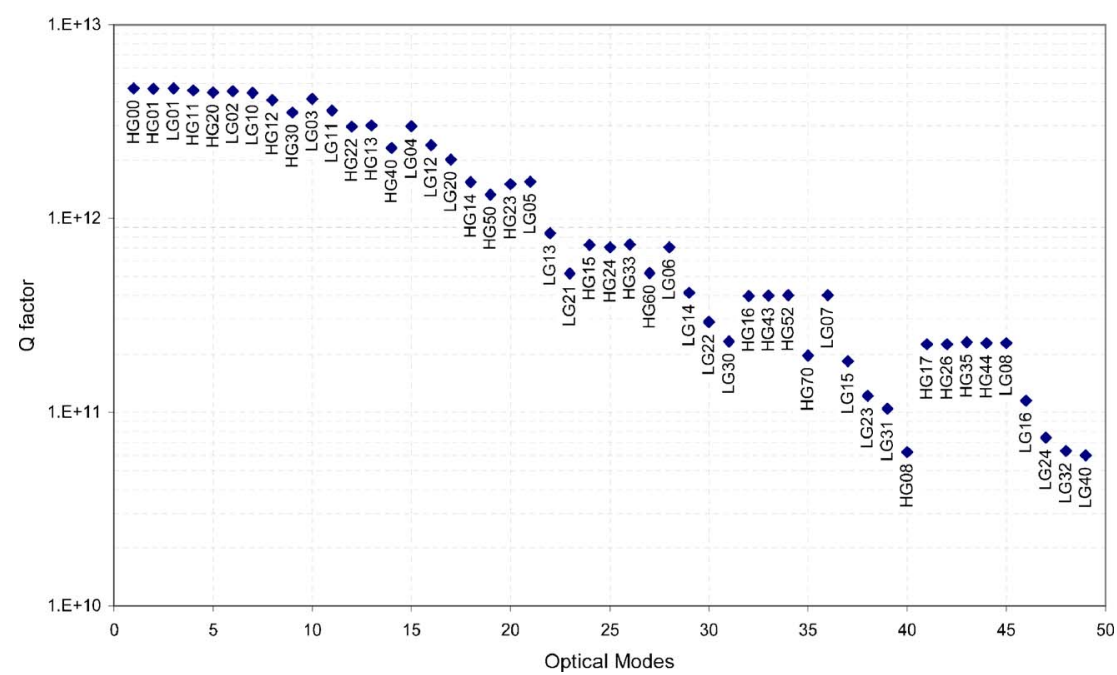

Fig. 10. (Color online) Optical $Q$ factor for the higher-order modes in the proposed cavity. 


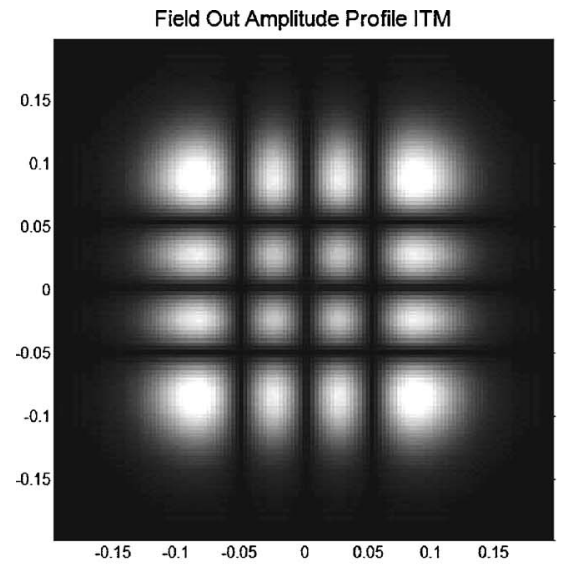

(a)

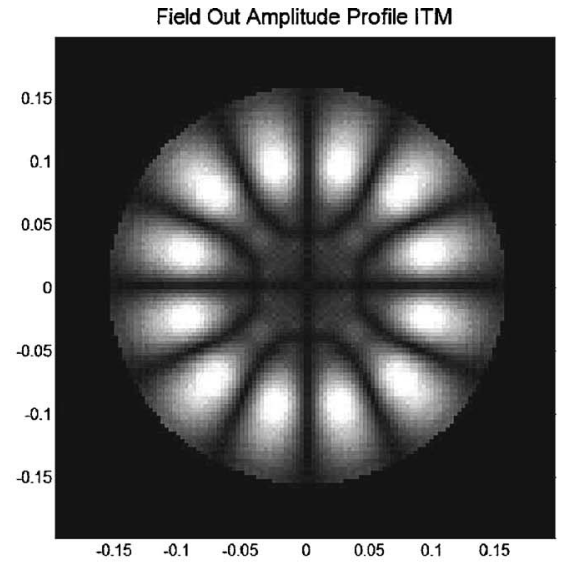

(b)

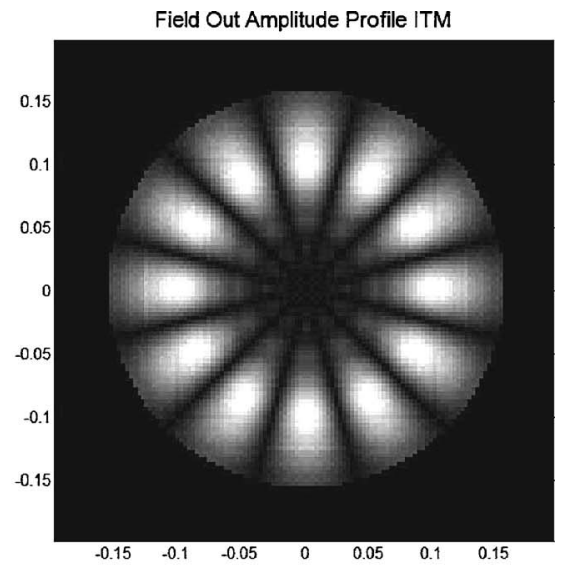

(c)

Fig. 11. (a) Intensity profile of mode HG33 in infinite-sized mirrors. (b), (c) Intensity profiles are for finite-sized mirrors of diameter $34 \mathrm{~cm}$. (b) The intensity profile of mode HG33 on the ITM at the end of the simulations. (c) The intensity profile of mode LG06 also for finite-sized mirrors.

optical modes will also have a dependency on the size of the mirrors, since both the frequency and the losses depend on the size of the mirror as well. Figure 10 shows the optical $Q$ factor for the higher-order modes for the $34 \mathrm{~cm}$ diameter mirrors. Here we can see that the $Q$ factor follows the same trend as in the optical gain, which also is inversely proportional to the total losses of the cavity.

\section{Mode Shape}

Higher-order modes have a more spread-out intensity profile. As a consequence, depending on the symmetry of the mode, it will cover a larger area of the mirror's surface. This not only causes high diffraction losses due to the energy loss per round trip but in some cases it also distorts the mode. The overlap parameter $\Lambda$ used to calculate the parametric gain $R_{0}$ depends on the mode distribution over the mirror's surface. ${ }^{3}$

An interesting case is mode HG33. This mode resonates and keeps its shape inside the cavity if the mirrors are of infinite size [Fig. 11(a)], but when finite-size mirrors are used the mode is completely distorted, and after a few round trips it does not look like a mode HG33 anymore but more like mode LG06, although twisted by $30 \mathrm{deg}$ and thus orthogonal to LG06, as can be seen in Fig. 11. The distorted mode HG33 still shows some features from the original mode such as the small energy distribution at the center of the intensity profile. However, it is easy to see the similarity and why the diffraction losses are higher for mode HG33 due to the energy loss at the edge of the mirror.

The interpretation is that even if we forced mode HG33 into a perfectly aligned cavity it will not resonate inside the cavity and will give rise to a twisted LG06 mode [Fig. 11(b)]. This mode will also be one of the eigenvectors of the cavity, since it is orthogonal to the original LG06 shown in Fig. 11(c). Similar effects were observed for modes HG43 and HG52.

\section{CONCLUSIONS}

We have shown how the diffraction losses of various modes in large optical cavities depend on the diameter of the mirrors. Moreover, they also depend on the shape of the mirrors. We show that the predicted mode frequencies are also offset from the infinite-mirror case by up to a few kilohertz. The diffraction losses are needed to determine the optical $Q$ factor of each mode, and this, combined with mode frequency data, is necessary to estimate the possibility of parametric instabilities, through the calculation of the parametric gain. We have shown that finite-size mirrors significantly alter the shape of the higher-order modes, and, due to high diffraction losses on each round trip, also the optical gain is reduced. The mode shape variations affect the overlap integral calculation, which determines the optoacoustic coupling in the parametric instability calculations.

We point out that in a power-recycled interferometer the design of the power-recycling cavity can vary the coupling losses so as to increase the high-order mode losses. The high-order losses can never be less than the diffraction losses predicted here. However, it is also true in the case of coupled cavities the mode shape could be significantly altered (compared with the single-cavity modes considered here), and this could vary the diffraction losses. The presence of a signal-recycling cavity can also affect the parametric instability.

\section{ACKNOWLEDGMENTS}

The authors thank Erika D'Ambrosio, Bill Kells, and Hiro Yamamoto for useful discussions. Also thanks to Jérôme Degallaix for his help in developing the FFT code at UWA and to Juri Agresti for his eigenvalue calculations. David G. Blair and Li Ju thank the LIGO Laboratory for its hospitality. Biplab Bhawal is supported by National Science Foundation under cooperative agreement PHY-0107417. This work was supported by the Australian Research Council and is part of the research program of the Australian Consortium for Interferometric Gravitational Astronomy.

Corresponding author Pablo Barriga can be reached at School of Physics M013, The University of Western Australia, 35 Stirling Highway, Crawley, Western Australia 
6009, Australia, or by phone, 61-8-6488-4552, or e-mail, pbarriga@cyllene.uwa.edu.au.

\section{REFERENCES}

1. V. B. Braginsky, S. E. Strigin, and S. P. Vyatchanin, "Parametric oscillatory instability in Fabry-Perot interferometer," Phys. Lett. A 287, 331-338 (2001).

2. W. Kells and E. D'Ambrosio, "Considerations on parametric instability in Fabry-Perot interferometer," Phys. Lett. A 299, 326-330 (2002).

3. C. Zhao, L. Ju, J. Degallaix, S. Gras, and D. G. Blair, "Parametric instabilities and their control in advanced interferometer gravitational-wave detectors," Phys. Rev. Lett. 94, 121102 (2005).

4. B. D. Cuthbertson, M. E. Tobar, E. N. Ivanov, and D. G. Blair, "Parametric back-action effects in a high- $Q$ cryogenic sapphire transducer," Rev. Sci. Instrum. 67, 2435-2442 (1996).

5. H. Rokhsari, T. Kippenberg, T. Carmon, and K. J. Vahala, "Radiation-pressure-driven micro-mechanical oscillator," Opt. Express 13, 5293-5301 (2005).

6. T. Corbitt, D. Ottaway, E. Innerhofer, J. Pelc, and N. Mavalvala, "Measurement of radiation-pressure-induced optomechanical dynamics in a suspended Fabry-Perot cavity," 0511022.pdf. http://arxiv.org/PS_cache/gr-qc/pdf/0511/

7. L. Ju, S. Gras, C. Zhao, J. Degallaix, and D. G. Blair, "Multiple modes contributions to parametric instabilities in advanced laser interferometer gravitational wave detectors," Phys. Lett. A 354, 360-365 (2006).

8. R. E. Spero, "Diffraction loss and minimum mirror size," LIGO Tech. Note, LIGO-T920002-00-D (LIGO, 1992).

9. R. E. Spero, "Update on diffraction loss and minimum mirror size," LIGO Tech. Note (LIGO, 2001).

10. E. D'Ambrosio, "Nonspherical mirrors to reduce thermoelastic noise in advanced gravitational wave interferometers," Phys. Rev. D 67, 102004 (2003).

11. A. G. Fox and T. Li, "Resonant modes in a maser interferometer," Bell Syst. Tech. J. 40, 453-488 (1961).

12. G. D. Boyd and J. P. Gordon, "Confocal multimode resonator for millimeter through optical wavelength masers," Bell Syst. Tech. J. 40, 489-508 (1961).

13. M. Born and E. Wolf, Principles of Optics, 7th ed. (Cambridge U. Press, 2003), pp. 412-516.

14. J. Y. Vinet, P. Hello, C. N. Man, and A. Brillet, "A high accuracy method for the simulation of non-ideal optical cavities," J. Phys. I 2, 1287-1303 (1992).

15. E. A. Sziklas and A. E. Siegman, "Diffraction calculations using fast Fourier transform methods," Proc. IEEE 62, 410-412 (1974).

16. B. Bhawal, "Diffraction losses of various modes in advanced LIGO arm cavity," LIGO Tech. Note, LIGOT050234-00-E (LIGO, 2005).

17. A. E. Siegman, Lasers (University Science, 1986), pp. $398-456$

18. R. Lawrence, "Active wavefront correction in laser interferometric gravitational wave detectors," Ph.D. thesis (Massachusetts Institute of Technology, 2003).

19. B. E. A. Saleh and M. C. Teich, Fundamentals of Photonics (Wiley, 1991) pp. 310-341.

20. A. E. Siegman, Lasers (University Science, 1986), pp. 626-662.

21. J. Agresti, LIGO Laboratory, California Institute of Technology, Pasadena, Calif. 91125, USA (private communication, 2005).

22. C. Yuanying, W. Youqing, H. Jin, and L. Jiarong, "An eigenvector method for optical field simulation," Opt. Commun. 234, 1-6 (2004)

23. J. P. Goldsborough, "Beat frequencies between modes of a concave-mirror optical resonator," Appl. Opt. 3, 267-275 (1964). 